\title{
TO TYPE OR TO PRINT? ONCOTYPE DX AND MAMMAPRINT TESTS FOR BREAST CANCER
}

\section{Hossain AZMM ${ }^{1}$}

Breast cancer is the most common cancer in women worldwide and it is the leading cause of death from cancer in women worldwide. ${ }^{1}$ Prognostic factors for the recurrence of breast cancer at a distant site regardless of treatment include clinicopathologic features such as tumor size and grade and the number of axillary lymph nodes with metastasis. ${ }^{2}$ Predictive factors that identify a benefit from specific therapies include the expression of the estrogen receptor and the progesterone receptor, which identifies patients who benefit from adjuvant endocrine therapy, ${ }^{3}$ and overexpression of the human epidermal growth factor receptor 2 (HER2) protein (or HER2 gene amplification), ${ }^{4}$ which identifies patients who benefit from adjuvant HER2-directed therapy.

Traditionally, breast oncology specialists have relied on clinical and pathologic tumor features as well as patient characteristics to determine risk of recurrence and to guide recommendations for adjuvant chemotherapy. However, such treatment decisions remain challenging, especially in patients with estrogen receptor-positive (ER-positive) early stage breast cancer where the absolute benefit of adjuvant chemotherapy is more difficult to perceive. Although studies have shown overall efficacy of adjuvant chemotherapy in the early stage breast cancer population, ${ }^{5}$ many patients are exposed to the associated risks of chemotherapy without deriving significant benefit from it.

Multiple molecular assays have been developed to help tailor therapeutic strategies for early stage breast cancer. ${ }^{6-7}$. One such assay is the Oncotype DX (Genomic Health, Redwood City, CA), a 21-gene breast cancer-specific expression profile which is now being utilized to improve patient selection for adjuvant chemotherapy. With use of a panel of 16 cancerrelated genes and 5 reference genes, the likelihood of developing distant recurrence in ER-positive early stage breast cancer is calculated as a recurrence score (RS) ranging from 0 to 100. The RS is based on the assumption that patients will receive adjuvant hormonal manipulation for 5 years and predicts a 10-year distant recurrence risk as a continuous variable. RS can also be subdivided into three risk categories: low $(<18)$, intermediate (18-30), and high $(>30)$ scores.

Study from a large multicenter prospective trial, the NSABP B-14 estimates of risk of distant recurrence at $6.8 \%, 14.3 \%$, and $30.5 \%$ with low, intermediate, and high RS, respectively. ${ }^{8}$ Since then, multiple studies worldwide have validated the prognostic capability of this assay. ${ }^{9-10}$ A subsequent study explored utilization of Oncotype DX categorical scores for prediction of additive benefit of chemotherapy to hormonal therapy. Patient tumor samples from another large multicenter prospective trial, the NSABP B-20 trial, which compared tamoxifen alone with chemotherapy plus tamoxifen treatment in node-negative, ER-positive, invasive breast cancer were assayed for RS. Patients with low RS $(<18)$ demonstrated no benefit from chemotherapy, whereas high scoring patients (RS $>31$ ) had maximum benefit (absolute benefit of 28\% reduction in distant metastatic disease development at 10 years) from adjuvant chemotherapy. ${ }^{11}$

More recently, the applicability of Oncotype DX $\mathrm{RS}$ both in node-positive breast cancer and in predicting local regional recurrence has been

1. AZM Mostaque Hossain, Professor \& Head, Department of Surgery, Dhaka Medical College \& Hospital 
studied. The TransATAC study found RS to be independently predictive of distant recurrence and thus prognostic in node-positive breast cancer patients. ${ }^{10} \mathrm{~A}$ retrospective analysis of a subset of node-positive patients in the SWOG8814 study has demonstrated that the multigene assay was prognostic and predictive of chemotherapy benefit. ${ }^{12}$ The Oncotype DX assay was also studied in the tamoxifen-treated patients from both the NSABP B-14 and B-20 trials to evaluate for association with local regional recurrence and revealed a significant association between RS and risk of local regional recurrence. ${ }^{12}$

In conclusion, the use of Oncotype DX assists in patient selection for adjuvant chemotherapy in breast cancer. Although there are multiple clinicopathologic features that correlate with RS, risk estimation based on traditionally used tumor characteristics results in a high rate of over- and underestimation of distant recurrence risk compared with Oncotype DX. Finally, the use of emerging nomograms aimed at replicating Oncotype DX may be of both clinical and economic utility in the future.

On the other hand MammaPrint test helps to estimate the risk of recurrence or distant metastases in early-stage breast cancer patients, whether their tumors are ER+ or ER.13-14 Thus, it has broader applicability than Oncotype DX. MammaPrint is performed in patients who are younger than age 61 years and have stage I or II, lymph node-negative cancer, with a tumor size of less than $5 \mathrm{~cm} .{ }^{15}$ It measures the expression of 70 genes in tumors-a lot more than Oncotype DX measures. MammaPrint classifies tumors as high- versus low risk of recurrence, and helps to decide if and what type of treatment is needed after surgery.

Because it is applicable for diverse types of breast cancer, MammaPrint may become more widely used once more evidence for its accuracy is available. The U.S. Food and Drug Administration (FDA) recently approved MammaPrint for testing of archival (paraffinembedded) tissues, which may broaden its applicability as well.

\section{References}

1. Jemal A, Center MM, DeSantis C, Ward EM. Global patterns of cancer incidence and mortality rates and trends. Cancer Epidemiol Biomarkers Prev 2010;19:1893-1907

Hayes D, Padnos SB. Predictive and prognostic markers in cancer. Clin Adv Hematol Oncol 2011;9:130-132

2. Hammond ME, Hayes DF, Dowsett M, et al. American Society of Clinical Oncology/College of American Pathologists guideline recommendations for immunohistochemical testing of estrogen and progesterone receptors in breast cancer. J.Clin.Oncol 2010;28:2784-2795

3. Wolff AC, Hammond ME, Hicks DG, et al. Recommendations for human epidermal growth factor receptor 2 testing in breast cancer: American Society of Clinical Oncology/College of American Pathologists clinical practice guideline update. J Clin Oncol 2013;31:3997-4013

4. EBCTCG. Effects of chemotherapy and hormonal therapy for early breast cancer on recurrence and 15-year survival: an overview of the randomised trials. Lancet. 2005;365(9472):1687-1717.

5. Turaga K, Acs G, Laronga C. Gene expression profiling in breast cancer. Cancer Control. 2010;17:177-182.

6. Ross JS, Hatzis C, Symmans WF, et al. Commercialized multigene predictors of clinical outcome for breast cancer. The Oncologist. 2008;13:477-493.

7. Paik S, Shak S, Tang G, et al. A multigene assay to predict recurrence of tamoxifen-treated, nodenegative breast cancer. N Engl J Med. 2004; 351:2817-2826.

8. Toi M, Iwata H, Yamanaka $\mathrm{T}$, et al. Clinical significance of the 21-gene signature (oncotype $\mathrm{dx}$ ) in hormone receptor-positive early stage primary breast cancer in the japanese population. Cancer. 2010;116:3112-3118.

9. Dowsett M, Cuzick J, Wale C, et al. Prediction of risk of distant recurrence using the 21 -gene recurrence score in node-negative and nodepositive postmenopausal patients with breast cancer treated with anastrozole or tamoxifen: A transatac study. J Clin Oncol. 2010;28:18291834.

10. Paik S, Tang G, Shak S, et al. Gene expression and benefit of chemotherapy in women with nodenegative, estrogen receptor-positive breast cancer. J Clin Oncol. 2006;24:3726-3734.

11. Albain KS, Barlow WE, Shak S, et al. Prognostic and predictive value of the 21 -gene recurrence score assay in postmenopausal women with node- 
positive, oestrogen-receptor-positive breast cancer on chemotherapy: a retrospective analysis of a randomised trial. Lancet Oncol. 2010;11:55-65.

12. Mamounas EP, Tang G, Fisher B, et al. Association between the 21-gene recurrence score assay and risk of locoregional recurrence in node-negative, estrogen receptor-positive breast cancer: results from nsabp b-14 and nsabp b-20. J Clin Oncol. 2010;28:1677-1683.

13. Mark F, Molecular Subtyping Identifies Chemosensitivity in Breast Cancer Patients
Conference Report | September 28, 2015 | ASCO 2015 Breast Cancer Symposium, Breast Cancer Targets

14. Chia SK, Bramwell VH, Tu D, et al. A 70-gene intrinsic subtype classifier for prognosis and prediction of benefit from adjuvant tamoxifen. Clin Cancer Res. 18(16):4465-4472, 2012.

15. Paik S, Shak S, Tang G, et al. A multigene assay to predict recurrence of tamoxifen-treated, nodenegative breast cancer. N Engl J Med. 30; 351(27):2817-26, 2004. 